\title{
Accurate simulation of Raman amplified lightwave synthesized frequency sweeper
}

\author{
Pedersen, Anders Tegtmeier; Olesen, Anders Sig; Rottwitt, Karsten
}

Published in:

Optical Society of America. Journal B: Optical Physics

Link to article, DOI:

10.1364/JOSAB.28.001493

Publication date:

2011

Document Version

Publisher's PDF, also known as Version of record

Link back to DTU Orbit

Citation (APA):

Pedersen, A. T., Olesen, A. S., \& Rottwitt, K. (2011). Accurate simulation of Raman amplified lightwave synthesized frequency sweeper. Optical Society of America. Journal B: Optical Physics, 28(6), 1493-1497. https://doi.org/10.1364/JOSAB.28.001493

\section{General rights}

Copyright and moral rights for the publications made accessible in the public portal are retained by the authors and/or other copyright owners and it is a condition of accessing publications that users recognise and abide by the legal requirements associated with these rights.

- Users may download and print one copy of any publication from the public portal for the purpose of private study or research.

- You may not further distribute the material or use it for any profit-making activity or commercial gain

- You may freely distribute the URL identifying the publication in the public portal 


\title{
Accurate simulation of Raman amplified lightwave synthesized frequency sweeper
}

\author{
Anders Tegtmeier Pedersen,* Anders Sig Olesen, and Karsten Rottwitt \\ DTU Fotonik, Technical University of Denmark, Ørsteds Plads 343, 2800 Kgs. Lyngby, Denmark \\ *Corresponding author: antp@fotonik.dtu.dk
}

Received February 1, 2011; revised April 12, 2011; accepted April 15, 2011;

posted April 15, 2011 (Doc. ID 140804); published May 20, 2011

\begin{abstract}
A lightwave synthesized frequency sweeper using a Raman amplifier for loss compensation is presented together with a numerical model capable of predicting the shape of individual pulses as well as the overall envelope of more than 100 pulses. The generated pulse envelope consists of 116 pulses with constant peak power and no significant growth of noise. The numerical simulation is based on careful measurements of the physical properties of the individual components and a well established Raman amplifier model. Very good agreement between the measured and the simulated data is found. (C) 2011 Optical Society of America

OCIS codes: $\quad 060.2630,140.3280,140.3550$.
\end{abstract}

\section{INTRODUCTION}

Throughout the last decade we have witnessed an increasing interest in frequency swept light sources due to their great potential within a variety of different applications. A promising version of such a source is the lightwave synthesized frequency sweeper (LSFS), which works by changing the carrier frequency in small discrete steps as a function of time. Since it was first described in 1990 [1] , it has been investigated in a number of publications where the main effort has been to maximize the sweeping range of the LSFS through clever use of sweeping filters, polarization control, etc. [2-4]. Potential applications of the LSFS cover many different areas, such as WDM systems [5] $]$, medical imaging []], dispersion measurement [7], and remote sensing of the atmosphere [8]. Theoretically the LSFS has successfully been described in [9], by assuming that the total power in the configuration used was constant at all times, and consequently the previously reported work was not capable of predicting the shape of individual pulses.

In [10] we experimentally demonstrated for the first time an LSFS using a Raman amplifier for loss compensation. In this work we present a numerical model of the LSFS that predicts both the shape of the individual pulses and the envelope of a train of pulses. In addition, we compare the results of the numerical model against detailed experimental measurements of the LSFS. The goal of the combined numerical and experimental effort is to design an LSFS that generates a high number of pulses in succession while maintaining a flat overall envelope.

\section{EXPERIMENTAL SETUP}

The experimental setup is shown in Fig. 1. It consists of a fiber optical ring with a Raman amplifier, a fixed bandpass filter (BPF) with an FWHM of $0.84 \mathrm{~nm}$, and an acousto-optic modulator (AOM). Light is coupled in and out of the ring through a $3 \mathrm{~dB}$ coupler. The Raman amplifier is a $3.17 \mathrm{~km}$ long high gain Raman fiber from OFS Fitel Denmark, backward pumped through a WDM coupler by a $1455 \mathrm{~nm}$ fiber laser. The Raman fiber has a gain coefficient of $2.44(\mathrm{~W} \cdot \mathrm{km})^{-1}$ at $1453 \mathrm{~nm}$ and attenuation of $0.40 \mathrm{~dB} / \mathrm{km}$ at $1450 \mathrm{~nm}$ and $0.31 \mathrm{~dB} / \mathrm{km}$ at $1550 \mathrm{~nm}$. An isolator heavily attenuates residual pump light and ensures one way circulation in the ring. To generate the first pulse, a $1565 \mathrm{~nm} \mathrm{CW}$ fiber laser with a linewidth of less than $10 \mathrm{kHz}$ from Koheras and an AOM were used. The resulting pulse train is monitored using an InGaAs photodetector and an oscilloscope.

The first pulse within the LSFS is created by modulation of the CW signal by the input AOM, and the coupler directs half of the pulse power to the photodetector and the other half into the ring. In the ring the pulse is amplified by the Raman amplifier and passes through the BPF, whereas spontaneous emission from the amplifier at wavelengths away from the signal wavelength is heavily attenuated, and noise in the ring is thereby limited. In the AOM inside the ring the frequency of the pulse is shifted $40 \mathrm{MHz}$. After a full revolution in the ring, the coupler again directs half of the pulse power to the output and the other half to the ring, where it is again amplified and frequency shifted $40 \mathrm{MHz}$. In this way an output that, over time, is swept in discrete steps of $40 \mathrm{MHz}$ is created. The pulse train is terminated by closing the $\mathrm{AOM}$ inside the ring.

To obtain a stable pulse train, two parameters are important to optimize. First of all, the gain of the amplifier should match the loss in the ring experienced by the pulses, thereby ensuring all pulses have the same output power. Second, the pulse length should not be longer than the round-trip time, because pulses will then leak into each other and consequently obscure the spectrum. However, by carefully matching the pulse length and the round-trip time, an output that is nearly constant in time and in which each pulse has only one distinct signal frequency is obtained. A limiting factor in the practical case is the finite closing time of the input AOM. The transmission is reduced by $90 \%$ in $120 \mathrm{~ns}$, but the time it takes to extinguish the last $10 \%$ of transmission is longer than $1 \mu \mathrm{s}$, and the transmission in this regime falls off exponentially. In order to prevent the tail of exponential decay from leaking into the following pulse, it is necessary to set the pulse length a few microseconds shorter than the ring round-trip time. It is noted that even though seeded by a laser, the ring does not 


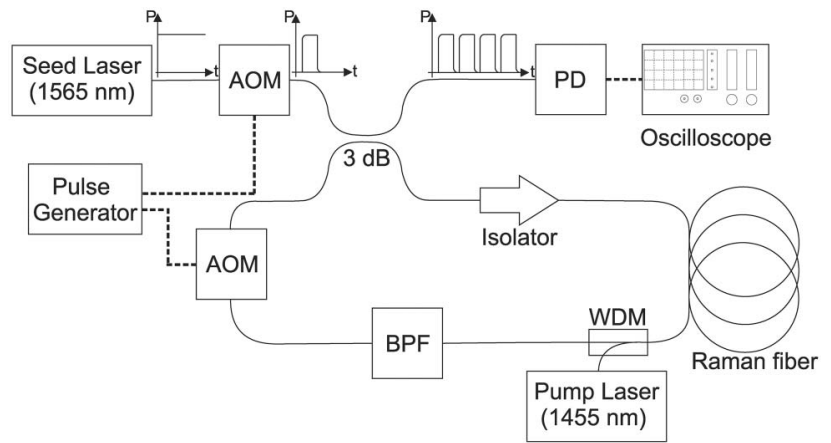

Fig. 1. Sketch of the experimental setup. Optical fibers are illustrated using full lines, and electrical wires, dashed lines. The inserts at the top are, from left to right, CW seed laser power, initial pulse modulated by the input AOM, and the resulting pulse train emitted from the LSFS.

operate as at laser cavity, since the frequency is shifted for each revolution.

\section{SIMULATION}

The approach in the numerical simulation is to include all physical effects that influence the LSFS and, thus, to neglect effects not affecting the LSFS. Dispersion is neglected due to the use of spectrally narrow pulses with a frequency bandwidth defined by the laser linewidth, and for the same reason the signal is simulated as monochromatic waves [11]. Because of the backward pumped Raman amplifier, the polarization dependence of the amplifier is not considered [12], and due to the frequency shift induced for each circulation of the ring and the down time between pulses, laser conditions are not required. Furthermore, stimulated Brillouin scattering, recaptured Rayleigh scattering, and backward propagating amplified spontaneous emission (ASE) are neglected due to the low power circulating in the ring at each frequency and the elimination of backward traveling light obtained by the isolator. Finally, scalar propagation equations are used.

In the simulation each component within the ring, except the fiber amplifier, is described by a transfer function in the time and frequency domains, respectively. The fiber amplifier is described using propagation equations. For each round trip of the ring, the signal power is propagated through each of the elements in the same order as in the physical ring. The $3 \mathrm{~dB}$ coupler, WDM coupler, and isolator are all simulated as a simple loss of power corresponding to the experimentally measured attenuation. The loss of the BPF is wavelength dependent and is fitted to the measured loss as a Gaussian function. The loss of the AOMs is simulated as a time dependent loss corresponding to the measured response function. The frequency shifts induced by the AOMs are simulated by defining the signal power as an array, representing monochromatic waves with a spacing equal to the shift induced by the AOMs $(40 \mathrm{MHz})$, and then shifting the entire array each time it passes through the AOM.

The Raman fiber amplifier is simulated by solving the dynamic propagation equations [12-14]

$$
-\frac{d P_{p}}{d z}+\frac{1}{V_{g}^{p}} \frac{d P_{p}}{d t}=-\sum_{i} \frac{\nu_{p}}{\nu_{s, i}} g_{R}\left(\nu_{p}-\nu_{s, i}\right) P_{P} P_{S, i}-\alpha_{p} P_{p}
$$

and

$$
\begin{aligned}
\frac{d P_{s, i}}{d z}+\frac{1}{V_{g}^{s}} \frac{d P_{s, i}}{d t}= & g_{R}\left(\nu_{p}-\nu_{s, i}\right) P_{p} P_{s, i}-\alpha_{s} P_{s, i} \\
& +2 g_{R}\left(\nu_{p}-\nu_{s, i}\right)[1+\eta(T)] h \nu_{s, i} B P_{p},
\end{aligned}
$$

where $z$ is the distance along the fiber, $t$ is the time, $V_{g}^{p, s}$ is the group velocity, $P_{p}$ is the pump power with frequency $\nu_{p}$, and $g_{R}$ is the frequency dependent Raman gain coefficient. $i$ is the frequency index of the signal power, $P_{s, i}$ is the signal power of frequency $\nu_{s, i}$, and $h$ is Planck's constant. $\alpha_{s}$ and $\alpha_{p}$ are the loss of the signal and pump, respectively. The signal loss is assumed to be the same for all signal frequencies. The first term on the right hand side of Eq. (1) accounts for the depletion of the pump due to the signal. In the following we demonstrate that this term has a significant impact on the pulse shape. The last term on the right hand side accounts for attenuation of the pump.

In Eq. (2) the first term on the right hand side describes gain, the second term attenuation, and finally the last term in Eq. (2) describes the spontaneous emission at the $i$ th frequency in a frequency bandwidth $B$ of $40 \mathrm{MHz}$ and the phonon occupancy factor

$$
\eta(T)=\frac{1}{\exp \left[\frac{h \Delta \nu}{k_{B} T}\right]-1},
$$

where $\Delta \nu$ is the frequency difference between signal and pump, $k_{B}$ is Boltzmann's constant, and $T$ is the absolute temperature.

The dynamic propagation equations [Eqs. (1) and (2)] are solved by discretization of the Raman fiber into sections $\Delta z$ and using a retarded time frame following signal and pump power by introducing the variables $\zeta^{+}=z+t / V_{g}$ and $\zeta^{-}=z-t / V_{g}$, respectively. The group velocity $V_{g}$ has here been assumed to be the same for all signal and pump frequencies. The equations then reduce to

$$
\frac{d P_{p}}{d \zeta^{-}}=\sum_{i} \frac{\nu_{p}}{\nu_{s, i}} g_{R}\left(\nu_{p}-\nu_{s, i}\right) P_{P} P_{S, i}+\alpha_{p} P_{p}
$$

and

$$
\begin{aligned}
\frac{d P_{s, i}}{d \zeta^{+}}= & g_{R}\left(\nu_{p}-\nu_{s, i}\right) P_{p} P_{s, i}-\alpha_{s} P_{s, i} \\
& +2 g_{R}\left(\nu_{p}-\nu_{s, i}\right)[1+\eta(T)] h \nu_{s, i} B P_{p} .
\end{aligned}
$$

By discretizing the Raman fiber and using the retarded time frame, the signal and pump powers are also discretized into time segments $\Delta t$ of corresponding lengths $\Delta t=\Delta z / V_{g}$. Equations (4) and (5) are solved for each spatial section and corresponding time segment for each power segment of the pulse train. The initial condition for the simulation is given by the steady state solution of Eqs. (1) and (2) at zero signal power.

The pulse train is generated by stepping through all time segments defined by the discretization of the Raman fiber. An array representing the power of the seed laser is predefined in time such that it generates the initial pulse and the leak power defined by the input AOM. For each time step the power from the seed laser array is added to the output of the ring at a corresponding time through the coupler and stepped through the individual components of the setup. The part of the array representing light leaking through the input AOM 
acts as the initial noise, which continues to grow due to the continuing addition of leaked light and spontaneous emission. The resulting power segment is then the output power at a time corresponding to the input time plus the travel time around the ring. The pulse train generated by the LSFS is a result of the modulated seed laser added with the output signal through the coupler for each time step.

\section{RESULTS}

An example of a measured pulse train consisting of 116 pulses, each $10 \mu \mathrm{s}$ long, a seed laser power of $5 \mathrm{~mW}$, and a pump laser power of $363 \mathrm{~mW}$ is shown in Fig. 2 , together with the corresponding simulation. The graph shown in the figure represents an average over 200 pulse trains. Figures 2(a)-2(c) show closeups of five pulses from the beginning, the middle, and the end of pulse train together with the simulation (dashed curve). The power has been normalized to the peak of the second pulse, since this is the first pulse that has propagated through the ring. The first pulses are clearly not rectangular, which is due to the finite response time of the input AOM. The pulse shape, however, rapidly changes, and in the middle of the train the power of the leading edge is stronger than the power of the trailing edge. This effect is due to depletion of the pump by the leading edge of the pulse, and because the pulse recirculates in the ring, the effect reinforces itself, leading to a larger and larger difference between the leading and the trailing edges of the pulses. This is seen in the last five pulses of the pulse train, where the leading edge has grown to be stronger than, not only the trailing edge, but also the preceding pulses, and the trailing edge has correspondingly dropped. The simulation is seen to predict the measured pulse train accurately. This agreement between measurement and simulation was only achieved when pump depletion was included in the simulations. Figure 2(d) shows a comparison of the envelopes of the measured (circles) and the simulated (solid curve) pulse train in their full lengths. Because the pulses become almost sawtooth shaped toward the end of the pulse train, four different points of comparison have been defined for each pulse: the peak, the mean pulse power, the trailing edge of each pulse, and the bottom level, defined as the mean power between the pulses. In the beginning the three graphs, representing the pulse powers, are seen to decay slightly, but after around $0.8 \mathrm{~ms}$, the mean power and the peak power start to rise, whereas the trailing edge continues to fall. For the mean power this behavior of falling and then rising is consistent with what has earlier been described in, e.g., [9]. Also, for this full length comparison, the simulation is seen to fit very well with the measurement, but with a slight deviation toward the end. It is noted that every other pulse is higher than the neighboring pulses. This phenomenon is especially pronounced for the peak of the pulses but is not found in the simulations.

Figures 2(a)-2(c) show a bottom level that is mainly due to the finite response time of the input AOM. However, from this level the buildup of ASE can be deduced, because an increase in the power level at the bottom must be attributed to the growth of ASE. In our pulse train the bottom level is flat around $5 \cdot 10^{-2}$ in the normalized units, and thus for this pulse train ASE does not grow significantly before the pulse train is terminated.

Figure 3 shows a pulse train in which the seed laser power has been increased to $11 \mathrm{~mW}$, while all other parameters have been kept constant as compared to the pulse train shown in Fig. 2. Again the power has been normalized to the peak of the second pulse. It is clear from the closeups both at the middle and at the end of the pulse train that the leading edge of the pulses grows up even more at the expense of the trailing edge than what was seen in Fig. 2. This is because of the shaping of the pulses caused by depletion of the pump, and with a higher signal this becomes more pronounced. In contrast to Fig. $\underline{2}$, the pulse power is seen to fluctuate more in the beginning of this pulse train than in the end. The simulation cannot

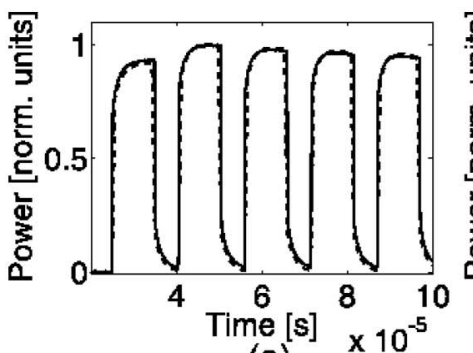

(a)

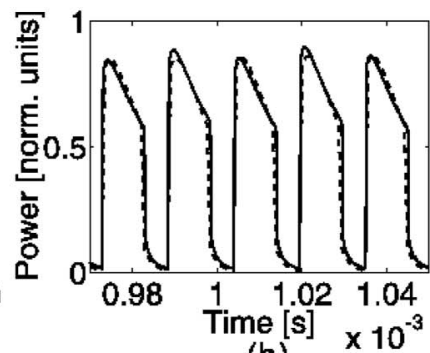

(b)

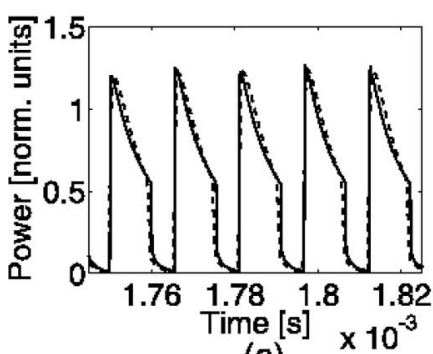

(c)

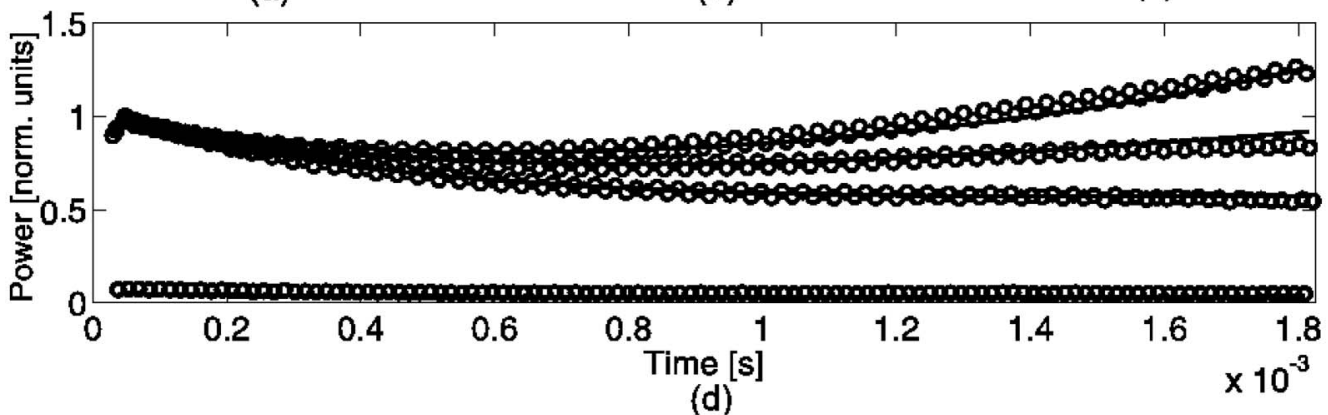

(d)

Fig. 2. Pulse train consisting of 116 pulses, a seed laser power of $5 \mathrm{~mW}$, and a pump laser power of $363 \mathrm{~mW}$. (a)-(c) Closeups of five pulses in the beginning, the middle, and the end of the pulse train. Solid curves represent measured data, and dashed curves, simulated data. (d) Full length comparison of measured and simulated data for the bottom, the trailing edge, the mean, and the peak of the pulses. Solid curves represent simulated data, and circles, measured data. 


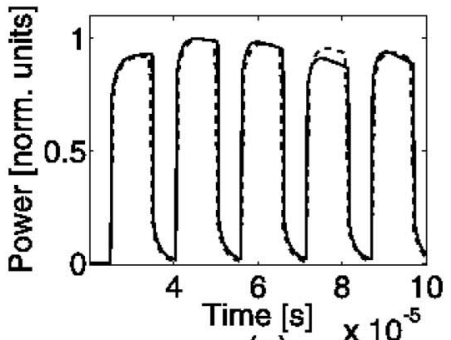

(a)

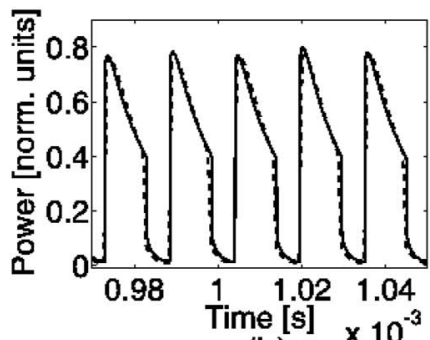

(b)

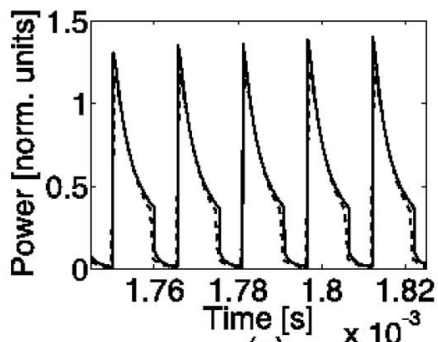

(c)

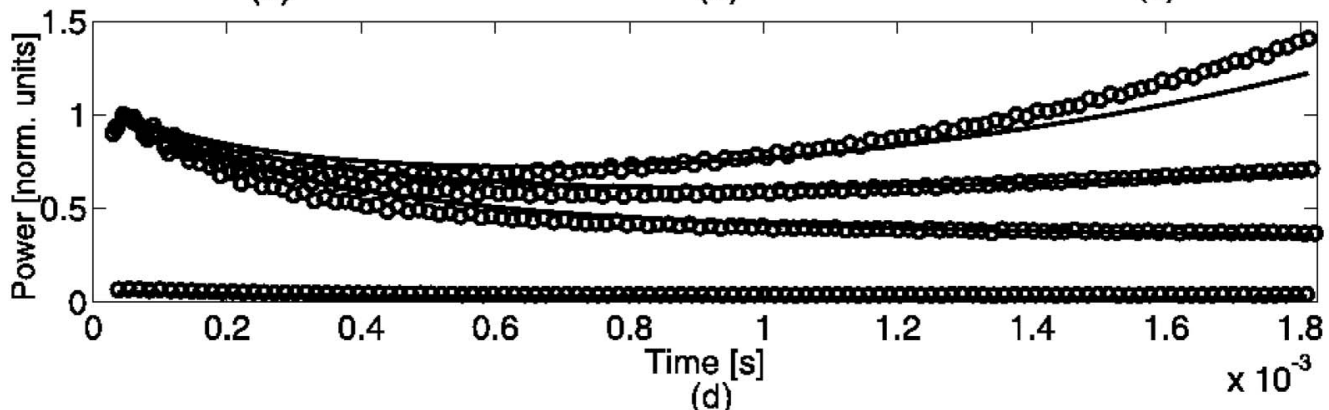

(d)

Fig. 3. Pulse train consisting of 116 pulses, a seed laser power of $11 \mathrm{~mW}$, and a pump laser power of $363 \mathrm{~mW}$. (a)-(c) Closeups of five pulses in the beginning, the middle, and the end of the pulse train. Solid curves represent measured data, and dashed curves, simulated data. (d) Pulse train envelopes for the bottom, the trailing edge, the mean, and the peak of the pulses. Solid curves represent simulated data, and circles, measured data.

replicate this, and the overall fit between measurement and simulation is not as good in the beginning of the pulse train as in the previous example. Toward the end of the pulse train the fluctuations become less pronounced, and the simulation agrees better with the measurements. However, in the end of the pulse train the shaping of the pulses becomes so strong that the simulation cannot reach the same peak power as the measurement, and a deviation between the measured and simulated pulse train is seen. Again the bottom level is flat, but here with a slightly lower value of around $4 \cdot 10^{-2}$ in the normalized units.

One of the goals of the work presented here has been to obtain as flat a pulse train as possible, and therefore the number of pulses has been limited to 116 . However, since noise does not seem to grow up in this case, there should be nothing to hinder an increase in the number of pulses generated at the expense of a less flat pulse train envelope. We predict that, perhaps in combination with a wider bandpass filter, generating pulse trains consisting of more than 500 pulses should be possible.

\section{CONCLUSION}

A lightwave synthesized frequency sweeper comprising a Raman amplifier for loss compensation has been demonstrated experimentally, and a numerical model capable of predicting the measured data has been developed. The LSFS generates a sequence of pulses in which the pulse rate is determined by the length of the ring and the number of pulses in one pulse train is determined by an acoustic modulator located inside the ring.

The numerical model accurately predicts the evolution of signals in the time domain. The model includes all components in the ring setup and their spectral characteristics. The input parameters for the model are deduced from careful measurements of each individual component in the LSFS. The output of the model is compared against experimental data, and excellent agreement is demonstrated.
The output of the ring has been optimized with respect to generating an output where the peak amplitude of each pulse is equal to the amplitude of the neighboring pulse. A pulse train with a flat envelope consisting of 116 individual pulses, each being $10 \mu \mathrm{s}$ long, has been demonstrated with no buildup of spontaneous emission. We have shown that the shape of the initial pulse is defined by the input acoustic modulator, but as the pulse train evolves, the shape of the pulses is eventually defined by the depletion of the amplifier pump. As a consequence, a large difference between the leading and trailing edges of the pulses is found. This effect seems unavoidable, and to the best of our knowledge there is no simple solution to this. The long-term stability of the setup has not been investigated in detail, but during operation no significant instabilities have been encountered. We believe that for long-term stability, stable seed laser and pump laser sources are the most critical.

\section{ACKNOWLEDGMENTS}

The Danish Agency for Science, Technology and Innovation, Research Infrastructure 2009, Grant No. 2136-08-0022, is thanked for financial support.

\section{REFERENCES}

1. P. Coppin and T. Hodgkinson, "Novel optical frequency comb synthesis using optical feedback," Electron. Lett. 26, 28-30 (1990).

2. H. Takesue and T. Horiguchi, "Broad-band lightwave synthesized frequency sweeper using synchronous filtering," J. Lightwave Technol. 22, 755-762 (2004).

3. H. Takesue, F. Yamamoto, K. Shimizu, and T. Horiguchi, " $1 \mathrm{THz}$ lightwave synthesised frequency sweeper with synchronously tuned bandpass filter," Electron. Lett. 34, 1507-1508 (1998).

4. H. Takesue, F. Yamamoto, K. Shimizu, and T. Horiguchi, "Stabilization of pulsed lightwave circulating around an amplified fiber-optic ring incorporating a Lyot depolarizer," IEEE Photon. Technol. Lett. 10, 1748-1750 (1998).

5. K. Aida and K. Nakagawa, "Time shared lightwave reference frequency distribution for photonics networks," J. Lightwave Technol. 14, 1153-1160 (1996). 
6. F. D. Nielsen, L. Thrane, P. E. Andersen, J. F. Black, and A. Bjarklev, "Swept wavelength source in the $1 \mu \mathrm{m}$ range," Opt. Express 13, 4096-4106 (2005).

7. H. Takesue and T. Horiguchi, "Chromatic dispersion measurement of optical components using lightwave synthesized frequency sweeper," J. Lightwave Technol. 20, 625-633 (2002).

8. P. Lindelöw and J. J. Mohr, "Coherent lidar modulated with frequency stepped pulse trains for unambiguous high duty cycle range and velocity sensing in the atmosphere," in IEEE International Geoscience and Remote Sensing Symposium, 2007 (IEEE, 2008), pp. 2787-2790.

9. K. Shimizu, T. Horiguchi, and Y. Koyamada, "Frequency translation of light waves by propagation around an optical ring circuit containing a frequency shifter: II. theoretical analysis," Appl. Opt. 33, 3209-3219 (1994).

10. A. T. Pedersen and K. Rottwitt, "Raman assisted lightwave synthesized frequency sweeper," in OpticalSensors, 2010 OSA Technical Digest (CD) (Optical Society of America, 2010), paper SWC5.

11. G. P. Agrawal, "Stimulated Raman Scattering," in Nonlinear Fiber Optics, 4th ed. (Academic, 2007), pp. 274-328.

12. K. Rottwitt and A. J. Stentz, "Raman amplification in lightwave communication systems," in Optical Fiber Telecommunication IV A (Academic, 2002), pp. 213-257.

13. J. Bromage, "Raman amplification for fiber communications systems," J. Lightwave Technol. 22, 79-93 (2004).

14. C.-J. Chen and W. S. Wong, "Transient effects in saturated Raman amplifiers," Electron. Lett. 37, 371-373 (2001). 